\title{
Immunohistochemical study of the brainstem cholinergic system in the alpaca (Lama pacos) and colocalization with CGRP
}

\author{
Pilar Marcos, ${ }^{1}$ Rafael Coveñas ${ }^{2,3}$ \\ ${ }^{1}$ Cellular Neuroanatomy and Molecular Chemistry of Central Nervous System, Faculty of Medicine, University of \\ Castilla-La Mancha, CRIB (Regional Centre of Biomedical Research), Albacete \\ ${ }^{2}$ Institute of Neurosciences of Castilla y León (INCYL), Laboratory of Neuroanatomy of the Peptidergic Systems, \\ University of Salamanca \\ ${ }^{3}$ Grupo GIR USAL: BMD (Bases Moleculares del Desarrollo), University of Salamanca, Spain
}

Several cholinergic regions have been detected in the brainstem of mammals. In general, these regions are constant among different species, and the nuclear complement is maintained in animals belonging to the same order. The cholinergic system of the brainstem has been partially described in Cetartiodactyla, except for the medulla oblongata. In this work carried out in the alpaca, the description of the cholinergic regions in this order is completed by the immunohistochemical detection of the enzyme choline acetyltransferase (ChAT). In addition, using double immunostaining techniques, the relationship between the cholinergic system and the distribution of calcitonin gene-related peptide (CGRP) previously described is analysed. Although these two substances are found in several brainstem regions, the coexistence in the same cell bodies was observed only in the laterodorsal tegmental nucleus, the nucleus ambiguus and the reticular formation. These results suggest that the interaction between ChAT and CGRP may be important in the regulation of voluntary movements, the control of rapid eye movement sleep and states of wakefulness as well as in reward mechanisms. Comparing the present results with others previously obtained by our group regarding the catecholaminergic system in the alpaca brainstem, it seems that CGRP may be more functionally related to the latter system than to the cholinergic system.

Key words: Alpaca; brainstem; calcitonin gene-related peptide; cholinergic system; immunohistochemistry.

Correspondence: Pilar Marcos, Cellular Neuroanatomy and Molecular Chemistry of Central Nervous System, Faculty of Medicine, University of Castilla-La Mancha, CRIB (Regional Centre of Biomedical Research), Avenida de Almansa 14, 02006 Albacete, Spain. E-mail: pilar.marcos@uclm.es

Contributions: The authors made an equal contribution: concept and design, immunohistochemistry, mapping, substantive intellectual contribution. The authors have read and approved the final version of the manuscript and agreed to be accountable for all aspects of the work.

Conflict of interest: The authors declare that they have no competing interests, and all authors confirm accuracy. 


\section{Introduction}

The alpaca (Lama pacos) is a member of the family Camelidae and belongs to the order Cetartiodactyla. This order includes cetaceans (whales and dolphins) as well as artiodactyls (eventoed ungulates), such as the giraffe and the sheep. ${ }^{1-5}$ The knowledge of the physiology of alpaca is important since it is part of an important sector of the economy of different countries in South America due to the excellent quality of its wool. ${ }^{6,7}$ The importance of this animal for the production sector has led studies of this species to focus on more practical aspects for its maintenance and reproduction, such as ovulation cycles or hormonal mechanisms. ${ }^{7}$ Although these studies are important on an economic perspective, the knowledge of the characteristics of these animals is interesting in itself. Alpacas have unique morphological (long neck with seven cervical vertebrae like other camelids ${ }^{8}$ ) and physiological characteristics since they are capable of living both at sea level and at 5,000 meters above sea level. ${ }^{7,9}$ This phenotype suggests the existence of fascinating adaptation mechanisms, mainly related to cardiovascular control, which must be regulated and controlled by the central nervous system. Other species included in Cetartiodactyla order also exhibit specific brain specializations in order to surviving within their respective environments. ${ }^{1-5}$

In this regard, several studies have been carried out on the alpaca brain reporting the distribution of neuroactive substances in the diencephalon ${ }^{9,10}$ and the brainstem. ${ }^{6,7,11}$ In the latter region, the distribution of leucine-enkephalin, ${ }^{6}$ calcitonin gene-related peptide $(\mathrm{CGRP})^{7}$ and the colocalization of CGRP with tyrosine hydroxylase (TH) have been described. ${ }^{11}$ The presence of the above-mentioned neuropeptides is widespread in the alpaca brainstem, and the distribution of TH (the limiting enzyme of catecholaminergic synthesis) showed that the nuclear parcellation of the catecholaminergic groups in the alpaca brainstem is coincident with the results described in other members of the same order. Previous studies have reported that species included in the same order exhibit the same nuclear complement in the neuromodulatory systems, supporting Manger's hypothesis. ${ }^{12}$ This affirmation has also been reported in the giraffe, a member of Artiodactyla order, where the organization of cholinergic, putative catecholaminergic and serotoninergic nuclei in the diencephalon, midbrain and pons has been described..$^{13,14}$ In addition, the organization of sleep-related neural systems has been described in other Cetartiodactyla such as Arabian oryx, ${ }^{1}$ minke whale, ${ }^{2}$ river hippopotamus, ${ }^{3}$ harbour porpoise, ${ }^{4}$ and blue wildebeest. ${ }^{5}$ Furthermore, the distribution of cholinergic structures has been reported in the sheep basal forebrain, ${ }^{15}$ but the distribution of cholinergic structures in the medulla oblongata of Artiodactyla remains unknown. In other mammalian species, including man, several cholinergic regions have been described in this part of the brainstem. ${ }^{14,16-37}$ For this purpose, one of the goals of this study is to map the location of the cholinergic structures in the alpaca medulla oblongata as well as in the other regions of the brainstem, and compare the results with that obtained in other mammals, including man.

Moreover, using double immunohistochemical methods, morphological relationships between CGRP and TH have been outlined in the alpaca brainstem that may be important in regions involved in cardiovascular control. ${ }^{9}$ The previous studies on the distributions of neuropeptides in the alpaca brainstem suggest that the location of CGRP-containing structures ${ }^{7}$ may be alike to that of cholinergic profiles described in other mammals. ${ }^{36}$ For instance, several cranial motor nuclei such as trochlear, motor trigeminal or facial displayed CGRP-immunoreactive neurons, ${ }^{7}$ and it has been reported that these motor nuclei and nerves are cholinergic. ${ }^{26}$ CGRP and acetylcholine are involved in functions such as motor control or nociception, suggesting that their anatomical localiza- tions may be similar. It has been reported that CGRP mRNA was detected in brainstem motoneurons in rat and guinea pig. ${ }^{18}$ In rats, the presence of CGRP in cholinergic structures of the brainstem such as the trigeminal complex has been previously reported, ${ }^{37}$ and coexistence of these two neuroactive substances has also been found in motoneurons of several cranial nuclei in cats. ${ }^{38}$ Thus, another aim of this study is to analyse the possible morphological relationships between the two neuroactive substances in the alpaca brainstem, by double immunohistochemical staining to detect CGRP and the enzyme choline acetyltransferase (ChAT), a marker for the cholinergic system. In addition, the distribution in the alpaca brainstem of the two neuromodulatory systems, cholinergic and putative catecholaminergic, will be compared. Thus, the mapping of the cholinergic system will be described for the first time in the alpaca brainstem as well as the neuroanatomical relationship between this system and CGRP. In the future, this study will serve to better understand the functional involvement of neuroactive substances in the altitude adaptation of alpacas and in their social and reproductive behaviour. In addition, this study can serve as a basis to develop future new works focused on the distribution and functions of classical neurotransmitters and neuropeptides in the alpaca central nervous system.

\section{Material and Methods}

\section{Animals}

As indicated in previous studies, ${ }^{7,9}$ adult male alpacas (Lama pacos $)(\mathrm{n}=5 ; 70-80 \mathrm{~kg} ; 5-8$ years $)$ maintained from birth to the perfusion at $0 \mathrm{~m}$ on the sea level were used. Procedures and protocols were carried out under the guidelines of the legal and ethics recommendations of the Spanish legislation. ${ }^{7}$ Alpacas had free access to water and food and were kept under standard conditions (temperature, light). ${ }^{7}$

\section{Tissue processing}

Alpacas were intravenously anaesthetized with xylazine (4 $\mathrm{mg} / \mathrm{kg})$ and ketamine $(10 \mathrm{mg} / \mathrm{kg})$ and perfused, through the carotid artery, with $3 \mathrm{~L}$ of $\mathrm{NaCl}(0.9 \%)$ and $5 \mathrm{~L}$ of paraformaldehyde $(4 \%)$ in phosphate-buffered saline (PBS, $0.15 \mathrm{M}, \mathrm{pH} 7.2) .{ }^{7}$ Brainstems were post-fixed overnight in the latter solution, cryoprotected with sucrose and frontal sections $(50-\mu \mathrm{m})$ were obtained using a cryostat. Sections were kept at $4^{\circ} \mathrm{C}$ in PBS. ${ }^{7}$

\section{Immunohistochemistry}

In this study, we used the same frontal sections in which CGRP-immunoreactivity was previously mapped applying the 33' diaminobenzidine (DAB) developing procedure. ${ }^{7}$ Thus, on these same frontal sections containing immunoreactivity for CGRP, a modified version of the protocol previously published (doublelabelling immunostaining) for the detection of ChAT was performed. ${ }^{39}$ Thus, free-floating frontal sections showing immunoreactivity for $\mathrm{CGRP}^{7}$ were treated with $\mathrm{H}_{2} \mathrm{O}_{2}(30 \%), \mathrm{NaOH}(1 \%)$ and $\mathrm{NH}_{3}(20 \%)$ to inactivate endogenous peroxidases. ${ }^{7}$ Then, sections were washed in PBS (6 x $10 \mathrm{~min})$ and pre-incubated in PBS containing Triton X-100 (0.3\%) and normal horse serum (1\%) (the latter solution was used for the dilution of antibodies and streptavidin). Sections were incubated overnight at $4^{\circ} \mathrm{C}$ with primary polyclonal antibody against ChAT (Millipore, ref. AB144 P) raised in goat and diluted 1:75; then washed in PBS ( $3 \times 10 \mathrm{~min})$ and incubated in biotinylated donkey anti-goat antibody (Jackson Laboratories) (room temperature, $90 \mathrm{~min}, 1 / 2,000)$. Later, sections were washed in PBS ( $3 \times 10 \mathrm{~min})$ and incubated in streptavidin coupled to peroxidase (Jackson Laboratories) (room temperature, 
90 min, 1/2,000). Subsequently, sections were washed in PBS (3 x $10 \mathrm{~min}$ ), Tris-HCl buffer (TB, $0.05 \mathrm{M}$; pH 7.6) and the product of the immunohistochemical reaction was revealed with 4-chloro-1napthol. This chromogen shows a blue precipitate which is easily distinguishable from the brown product due to the DAB used previously for the CGRP detection. Because the chloronaphtol reaction does not allow dehydration, sections were mounted on gelatine-coated slides and coverslipped with PBS/glycerol (1/3). To check the specificity of the ChAT immunohistochemical procedure, histological controls were performed by: 1) omission of the antibody in the first incubation bath and 2) replacing the secondary biotinylated antibody by PBS. In both cases, no immunoreactivity was observed. As previously described the specificity of the CGRP antiserum was also ckecked: ${ }^{7}$ 1) CGRP antiserum was preabsorbed with synthetic CGRP; 2) the first antibody was omitted in the first incubation bath; 3) the secondary biotinylated antibody was replaced by PBS; and 4) the CGRP antiserum was preabsorbed with an excess of related peptides (neuropeptide $Y$, vasoactive intestinal polypeptide, substance $\mathrm{P}$, calcitonin, amylin). The histological controls demonstrated the specificity of the immunoreactivity for CGRP. ${ }^{7}$ The distribution of the catecholaminergic and cholinergic systems in the alpaca brainstem was compared according to a previous published work focused on the mapping of TH. ${ }^{9}$

\section{Mapping}

As the main reference, the mapping of de Souza et al..$^{6,7}$ was followed for nomenclature and the location of the cholinergic groups in the alpaca brainstem, but in addition the distribution of these cell groups in other mammalian species was also consulted. ${ }^{1-}$ 5,8,14,17,21,23-28,30,32,36,37,40,41 The computerized digital mapping system Accustage MDPlot v5.2 (MD3-Digitizer, Accustage, Minnesota Datametrics, USA) was used to study the distribution of the immunoreactive neurons. Photographs were obtained with a digital camera attached to a Nikon Eclipse 80i microscope and using the Adobe Photograph 6.0 Software only the contrast and brightness of the images were adjusted.

\section{Results}

The distribution of the CGRP- and ChAT-immunoreactive structures has been mapped according to the anatomical description carried out by de Souza et al. ${ }^{6,7}$ Mapping of the structures containing CGRP (fibres and cell bodies) has previously been performed in the alpaca brainstem. ${ }^{7}$ Thus, the present work will be focused on the cholinergic profiles and on the coexistence of ChAT and CGRP in nerve cells. The brown precipitate corresponding to CGRP and the blue staining for ChAT are easily distinguishable. In addition, CGRP-immunoreactive fibres showed a varicose appearance and CGRP-positive cell bodies contained secretion granules typically peptidergic, whereas profiles containing the enzyme displayed a more homogeneous precipitate and no varicose labelling.

Since the description of pontomesencephalic cholinergic nuclei has been extensively described in other species included in the Cetartiodactyla order, ${ }^{1-5,14}$ this study will report mainly the cholinergic profiles observed in the medulla oblongata of the alpaca. Figure 1 shows the distribution patterns of ChAT and CGRP in the alpaca brainstem. In general, a caudo-rostral increase in the immunolabeling for ChAT was detected throughout the alpaca brainstem (Figure 1). In the most caudal levels of the medulla oblongata (Figure $1 \mathrm{H}, \mathrm{I}$ ), scarce neurons and fibres containing CGRP (Figure 2A) were observed mixed with ChAT-positive profiles in the nucleus ambiguus (Figure $2 \mathrm{~B}, \mathrm{C}$ ), nucleus of the hypoglossal nerve (XII), and the reticular formation. In the lateral reticular nucleus, isolated labelled neurons and a low density of fibres containing ChAT was observed. More rostrally, ChATimmunoreactive cell bodies and fibres were detected in the dorsal motor nucleus of the vagus (Figure $1 \mathrm{~F}, \mathrm{G}$ ) as well as in the cranial nerves and nuclei facial, abducens, and motor trigeminal (Figures $1 \mathrm{D}, \mathrm{E}$ and Figure $2 \mathrm{D}, \mathrm{E})$. Although the reticular formation displayed neurons containing ChAT and CGRP (Figure 1 and Figure $2 \mathrm{~F}$ ), only isolated double labelled cell bodies CGRP-ChAT were detected in the reticular formation as well as in the nucleus ambiguus (Figure 2 A,B,C,F).

As it has been observed in the giraffe, ${ }^{14}$ cranial nerve motor nuclei located in the alpaca medulla oblongata and pons displayed cholinergic cell bodies and fibers, and the cranial nerves themselves showed ChAT-immunoreactivity (Figure 2 D,E). These nuclei also contained cell bodies with CGRP, but no double labelled neurons were detected except for the nucleus ambiguus.

The pontine and mesencephalic reticular formation displayed neurons and fibers containing ChAT, mixed with CGRP-positive fibres. The location of the cholinergic cell groups in the pons and mesencephalon is similar to that described previously in the giraffe ${ }^{14}$ as well as in other members of Cetartiodactyla order. ${ }^{1-5}$ In the lateral pontine tegmentum, ventral to the anterior aspect of the inferior colliculus, ChAT-immunoreactive cell bodies and fibres were detected in the parabigeminal nucleus (Figure 1B). This is homologous to the $\mathrm{Ch} 8$ cell group in the human brainstem. ${ }^{27}$ Cell bodies and fibres containing CGRP were also found in this nucleus. In the ventrolateral portion of the periaqueductal and periventricular grey matter and caudal to the oculomotor nucleus, the laterodorsal tegmental nucleus (the Ch6 cholinergic cell group) displayed CGRP-positive cell bodies (Figure 1B and Figure 3A). Cholinergic neurons and fibres of the laterodorsal tegmental nucleus were detected in close proximity to CGRP-immunoreactive cell bodies and fibers, and double labelled structures were observed in this nucleus (Figure 1B and Figure 3 B,C). Similar to the giraffe ${ }^{14}$ and other Cetartiodactyla such as the hippopotamus and the blue wildebeest, ${ }^{3-5}$ the cholinergic cell bodies observed in the laterodorsal tegmental nucleus were slightly larger than the neurons of the pedunculopontine tegmental nucleus. In this nucleus, located within the dorsal aspect of the pontine tegmentum immediately inferior to the superior cerebellar peduncle and homologous to the $\mathrm{Ch} 5$ cholinergic cell group, numerous cell bodies and fibres positive for ChAT were detected (Figure 1B), mixed with CGRP-immunoreactive fibres (Figure 3D). Cholinergic neurons, extremely immunostained, were found in the pedunculopontine tegmental nucleus from the level of the oculomotor nucleus to the anterior limit of the trigeminal motor nucleus, mixed with neurons containing CGRP, which are very sparse in this area.

Cranial nerves and nuclei located in this part of the brainstem displayed abundant ChAT immunoreaction (Figure $1 \mathrm{~A}, \mathrm{~B})$, but scarce CGRP-positive neurons. In the trochlear (Figure 1B) and oculomotor (Figure 1A) nuclei, cholinergic cell bodies showed the typical morphology of motoneurons. As in the giraffe, ${ }^{14}$ the Edinger-Wesphal nucleus of the alpaca showed a staining for ChAT-immunoreactive neurons (Figure 1A).

\section{Discussion}

In this study the mapping of the cholinergic system has been described in the alpaca brainstem, thus completing the distribution pattern of the cholinergic system of Cetartiodactyla; in addition, the neuroanatomical relationship between this system and CGRP is also reported. This study will serve to develop future works focused on the distribution and functions of classical neurotransmitters and neuropeptides in the alpaca central nervous system. Moreover, this and previous ${ }^{6,9,10,11}$ studies will contribute to better 
understand the functional involvement of neuroactive substances in the altitude adaptation of alpacas and in their social and reproductive behaviour.

Despite being animals belonging to very different orders, there is a common cholinergic staining pattern in the brainstem that is conserved in giraffe, rat, mouse, guinea pig, cat, ferret, several megachiropterans and microchiropterans, common marmoset, squirrel monkey, macaque, baboons and humans ${ }^{13,14,16,17,19-37,40}$ which has been also observed in the alpaca brainstem. This common pattern includes the parabigeminal nucleus, the pedunculopontine and laterodorsal tegmental nuclei, and the nuclei of the motor cranial nerves III, IV, Vm, VI, VII, X, XII; as well as the nucleus ambiguus. All these regions also contained ChAT mRNA in the mouse brainstem, ${ }^{42}$ as well as in humans. ${ }^{31}$ On the other hand, other brainstem nuclei displayed ChAT immunostaining in other mammals, but not in the alpaca. For instance, peri-locus coeruleus cholinergic neurons were observed in the cat brainstem ${ }^{32}$ and the interpeduncular nucleus of the rat showed ChATimmunoreactive cell bodies..$^{21,24,36}$ In the guinea pig $^{28}$ and ferret, ${ }^{20}$ cholinergic cell bodies were detected in the superior colliculus or the parabrachial nuclei, which were devoid of ChAT immunostaining in the alpaca.

The distribution of the cholinergic profiles observed in the mesencephalon and pons of alpaca is in agreement with that reported in the giraffe, another member of the Artiodactyla order ${ }^{14}$ as well as in other Cetartiodactyla species, ${ }^{1-5,14}$ supporting the Manger's hypothesis that changes in the complexity of neural systems do not occur during speciation within an order irrespective to brain size, lifestyle and phenotype. ${ }^{12}$ Other works about the cholinergic system of Artiodactyla described the structures located in the basal forebrain ${ }^{15}$ or the olfactory bulb, ${ }^{44}$ but not the brainstem. The present paper describes the location of the cholinergic structures in the medulla oblongata, thus completing the mapping of the cholinergic system in the brainstem of Artiodactyla. In this sense, the distribution of ChAT in the medulla oblongata of the alpaca is similar to that found in other mammals, since the immunostaining was detected in the nuclei of the cranial nerves X and XII as well as the nucleus ambiguus, with both somatic and special visceral efferent components, and the reticular formation. ${ }^{13,16,17,19-37,40}$

In several regions of the alpaca brainstem, colocalization of ChAT and CGRP has been detected: nucleus ambiguus, reticular formation, facial nucleus, parabigeminal nucleus, pedunculopontine tegmental nucleus, and laterodorsal tegmental nucleus. However, coexistence of ChAT and CGRP in the same cell bodies has been observed only in the nucleus ambiguus and more frequently in the laterodorsal tegmental nucleus, and occasionally in the reticular formation. This limited coexistence could be due to methodological factors. It is important to note, as it has been previously described, ${ }^{39}$ that the product of the DAB reaction masks catalytic sites and the antigen of the first sequence of immunoreagents and hence the interaction with the reagents of the second sequence is prevented. However, in agreement with our results, other studies have also described a scarce coexistence of these two substances ChAT and CGRP in the brainstem of mammals. Coexistence has been reported in cells of the rat trigeminal ganglion, and it has been suggested that they might participate in nociception. ${ }^{37}$ CGRP mRNA has been detected in spinal and brainstem motoneurons of rat and guinea pig. ${ }^{18}$ In the cat brainstem, the majority of motoneurons containing ChAT located in somatomotor or branchiomotor cranial nuclei (VI, VII and XII nerves) as well as those of nucleus ambiguus are also CGRP-positive, and cell bodies containing CGRP observed in the ventromedial reticular formation, lateral cuneate nucleus, infratrigeminal nucleus and nucleus of the solitary tract are ChAT-negative..$^{38}$ Our results display a more restricted pattern of coexistence of CGRP and ChAT, and suggest that the interaction of CGRP and ChAT in the same neurons may be important in the projections from the laterodorsal tegmental nucleus (the homologue of Ch6 cholinergic group in humans) to subcortical structures such as the thalamus, the neostriatum or other mesopontine areas, ${ }^{30}$ implicated in the control of rapid eye movement (REM) sleep and states of wakefulness. ${ }^{25,27,40}$ Several studies carried out in different species of Cetardiodactyla ${ }^{1-5}$ and in cats $^{35}$ to analyse the neural systems implicated in the sleep regula-
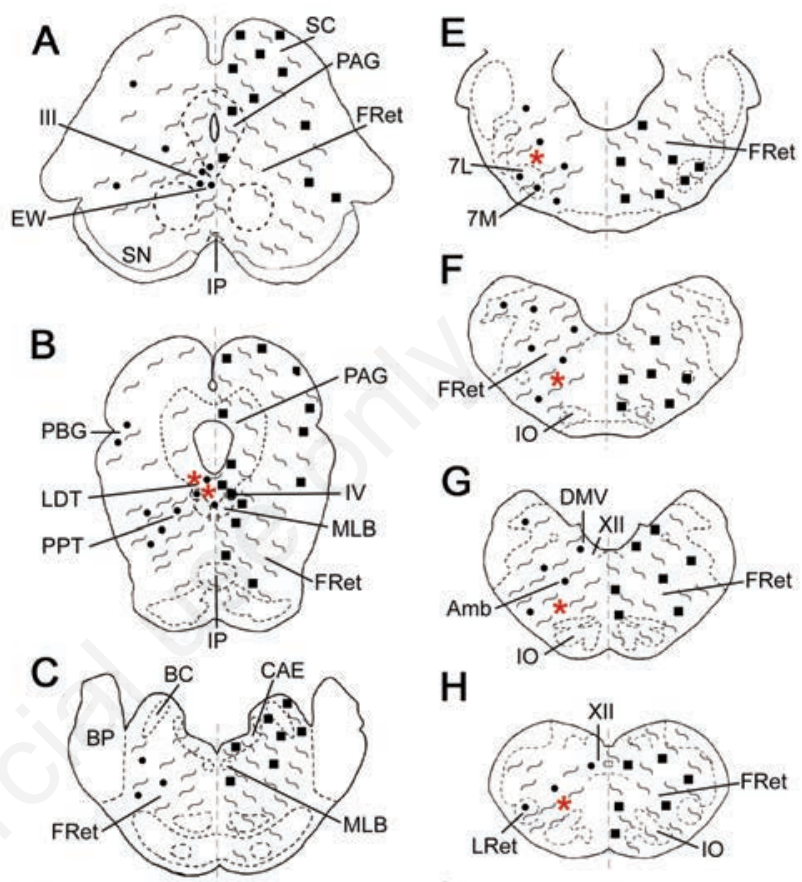

$\mathrm{H}$
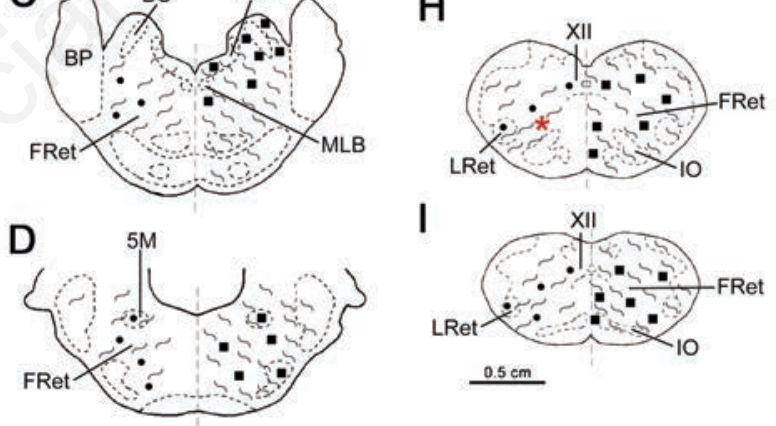

I

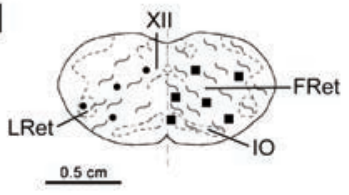

Figure 1. Mapping of the distribution of CGRP- and ChATimmunoreactive structures in the alpaca brainstem according to the anatomical description carried out by de Souza et al., ${ }^{6,7}$ from rostral (A) to caudal levels (I). For the detailed distribution of structures containing CGRP see de Souza et al. ${ }^{7}$ Mapping of CGRP is represented on the right side by squares and ChAT-positive structures are represented by dots on the left side, as well as double-labelled cell bodies (red asterisks). Circles show only location and are not reflective of actual numbers of positive neurons. Immunoreactive fibers are represented by continuous short lines. III, nucleus of oculomotor nerve (III cranial nerve); IV, nucleus of the trochlear nerve (IV cranial nerve); Vm, motor trigeminal nerve; VI, abducens nerve (VI cranial nerve); VII, facial nerve (VII cranial nerve); XII, nucleus of the hypoglossal nerve (XII cranial nerve); $5 \mathrm{M}$, motor trigeminal nucleus; $7 \mathrm{~L}$, facial nucleus, lateral division; 7M, facial nucleus, medial division; Amb, nucleus ambiguus; $\mathrm{BC}$, brachium conjunctivum; $\mathrm{BP}$, brachium pontis; CAE, locus coeruleus; DMV, motor dorsal nucleus of the vagus nerve; DRM, dorsal nucleus of the raphe; EW. nucleus of Edinger-Westphal; FRet, reticular formation; IO, inferior olive; IP, interpeduncular nucleus; LDT, laterodorsal tegmental nucleus; LRet, lateral reticular nucleus; MLB, medial longitudinal bundle; PAG, periaqueductal gray; PBG, parabigeminal nucleus; PPT, pedunculopontine tegmental nucleus; SC, superior colliculus; $\mathrm{SN}$, substantia nigra. 
tion reported that the cholinergic neurons of the laterodorsal tegmental nucleus may play an important role in initiating forebrain/neural aspects of REM sleep. ${ }^{5}$ The laterodorsal tegmental nucleus also modulates reward, since this nucleus preferentially targets circuits associated with limbic functions. ${ }^{44}$ The results obtained in the present work suggest that the interaction of CGRP/ChAT in neurons of the laterodorsal tegmental nucleus might be also implicated in the regulation of these functions.

The morphological characteristics of the laterodorsal tegmental nucleus and the pedunculopontine nucleus have been extensively described in Cetartiodactyla. ${ }^{1-5,14}$ These two nuclei are key sleepwake cycle nuclei specific to Artiodactyls and modulate the initiation of REM sleep but not REM sleep maintenance. ${ }^{1}$ Although their nuclear organization is consistent across the different species of Cetartiodactyla, these cholinergic nuclei display variability of their relative perikarya size that has not been reported in other mammalian species. ${ }^{4,5}$ For example, it has been described that the cholinergic cell bodies observed in the laterodorsal tegmental nucleus were slightly larger than the neurons of the pedunculopontine tegmental nucleus in the giraffe,,${ }^{14}$ the hippopotamus and the blue wildebeest, ${ }^{3-5}$ and this has been also detected in the alpaca. The opposite has been observed in the minipig and the Arabian oryx,${ }^{1}$ whereas in aquatic Cetartiodactyla the size of the cholinergic neurons detected in those nuclei was similar., ${ }^{2,4}$ These differences may be related to the different REM sleep initiation patterns showed by these animals. ${ }^{5}$

Although the methodology performed in this work does not reveal if mechanisms of co-transmission of the two substances exist, the results obtained suggest that in the alpaca brainstem the interaction between CGRP and ChAT in the same cell bodies (coexistence in two brainstem nuclei) is limited compared to the interaction between CGRP and the catecholaminergic system (particularly, noradrenaline), since seven of the 18 TH-immunoreactive regions of the alpaca brainstem showed double-labelled cell
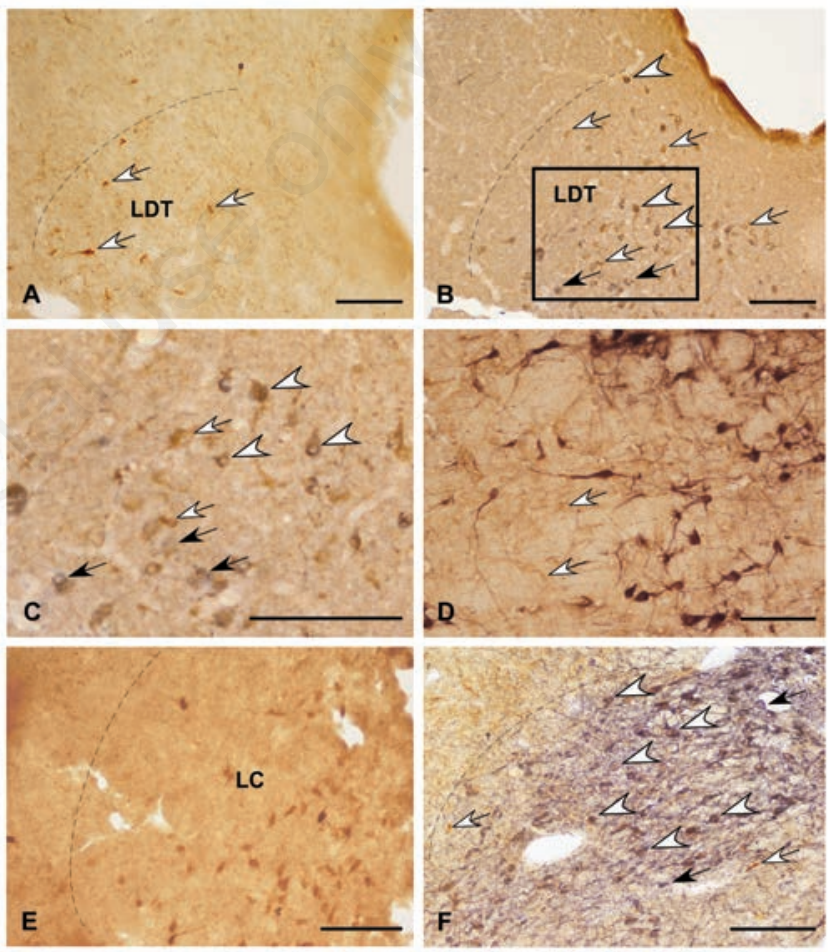

Figure 3. Pictures of different pontomesencephalic regions containing ChAT (blue precipitate) and CGRP (brown). The boundaries of each nucleus are delineated by dashed lines. A) Cell bodies containing CGRP in the laterodorsal tegmental nucleus (LDT). B) Cell bodies containing CGRP (white arrows) detected in the cholinergic LDT; numerous cholinergic cell bodies were observed (black arrows), as well as some neurons containing CGRP and ChAT (white arrowheads). C) High-power magnification of the area squared in B. Cholinergic cell bodies are pointed by black arrows and CGRP-positive neurons by white arrows; double-labelled cell bodies (white arrowheads) were also detected. D) High density, heavily immunostained cholinergic neurons observed in the pedunculopontine nucleus; scarce neurons containing CGRP (white arrows) are observed in this region. E) Noncholinergic CGRP-immunoreactive neurons observed in the locus coeruleus (LC). F) The same region displaying double labelling CGRP/TH (see text); massive coexistence (white arrowheads) can be observed in neurons of the LC for these two substances compared to CGRP/ChAT (E); some single-immunolabeled neurons are also detected (black arrow for $\mathrm{TH}$; white arrow for CGRP). Scale bar: $100 \mu \mathrm{m}$. 
bodies for CGRP and TH (compare Figure 3 panel E and panel F). These regions are $\mathrm{A} 1, \mathrm{C} 1, \mathrm{C} 2, \mathrm{~A} 6 \mathrm{~d}, \mathrm{~A} 7 \mathrm{sc}, \mathrm{A} 7 \mathrm{~d}$ and $\mathrm{A} 91$, and such interaction in these catecholaminergic areas might be important in the regulation of cardiovascular functions. ${ }^{9}$ However, it cannot be ruled out that CGRP and ChAT may also interact by means of volume transmission mechanisms, as it has been suggested for neurokinin and ChAT in the human brainstem. ${ }^{26}$ An overlap in the distribution of the catecholaminergic and cholinergic systems, but no cell bodies immunostained for both TH and ChAT, has been observed in the brainstem of the baboon, ${ }^{34}$ ferret ${ }^{19}$ and cat. ${ }^{22}$ This seems to be also the case in the alpaca brainstem and might contribute to explain the low degree of coexistence of CGRP and ChAT compared to CGRP and TH in this region.

In conclusion, the data provided in this work complement the mapping of the cholinergic system in the brainstem of Cetartiodactyla. The nuclear delineation is the same as that reported in other members of this order and is also similar to that described in other mammals. Despite the existence of morphological relationships between CGRP and ChAT, double-labelled neurons can be observed in a few regions. On the contrary, it seems that CGRP would be more functionally related to the catecholaminergic system than to the cholinergic system, since more nuclei with double immunostaining for CGRP and TH have been detected in the alpaca brainstem.

\section{Acknowledgements}

This work was supported by the "Programa XI: Financiación de Unidades de Excelencia de la Universidad de Salamanca" (Spain), DGICYT (PB99/0160), Ministerio de Ciencia y Tecnología (BFI2001-1905) and Junta de Comunidades de Castilla-La Mancha (Grants PAI-05-067 and PI-2006/14), Spain. Authors wish to thank the technical assistance of Mr. G. Lozano for his help with mapping and the Language Service (University of Salamanca, Salamanca, Spain) for supervising the English text.

\section{References}

1. Davimes JG, Alagaili AN, Bennett NC, Mohammed OB, Bhagwandin A, Manger PR, et al. Neurochemical organization and morphology of the sleep related nuclei in the brain of the Arabian oryx, Oryx leucoryx. J Chem Neuroanat 2017;81:5370.

2. Dell LA, Karlsson KA, Patzke N, Spocter MA, Siegel JM, Manger PR. Organization of the sleep-related neural systems in the brain of the minke whale (Balaenoptera acutorostrata). J Comp Neurol 2015;524:2018-35.

3. Dell LA, Patzke N, Spocter MA, Bertelsen MF, Siegel JM, Manger PR. Organization of the sleep-related neural systems in the brain of the river hippopotamus (Hippopotamus amphibius): a most inusual Certiodactyl species. J Comp Neurol 2015;524:2036-58.

4. Dell LA, Patzke N, Spocter MA, Siegel JM, Manger PR. Organization of the sleep-related neural systems in the brain of the harbour porpoise (Phocoena phocoena). J Comp Neurol 2016;524:1999-2017.

5. Malungo IB, Gravett N, Bhagwandin A, Davimes JG, Manger PR. A preliminary description of the sleep-related neural systems in the brain of the blue wildebeest, Connochaetes taurinus. Anat Rec 2020;303:1977-97.

6. De Souza E, Yi P, Aguilar LA, Coveñas R, Lerma L, Andrade $\mathrm{R}$, et al. Mapping of leucine-encephalin in the alpaca (Lama pacos) brainstem. In: Coveñas R, Mangas A, Narváez JA, editors. Focus on neuropeptide research. Trivandrum: Transworld Research Network; 2007. p. 103-13.

7. De Souza E, Coveñas R, Yi P, Aguilar LA, Lerma L, Andrade $\mathrm{R}$, et al. Mapping of CGRP in the alpaca (Lama pacos) brainstem. J Chem Neuroanat 2008;35:346-55.

8. Badlangana NL, Bhagwandin A, Fuxe K, Manger PR. Observations on the giraffe central nervous system related to the corticospinal tract, motor cortex and spinal cord: what difference does a long neck make? Neuroscience 2007;148:52234.

9. Marcos P, Arroyo-Jiménez MM, Lozano G, Aguilar LA, Coveñas R. Mapping of tyrosine hydroxylase in the alpaca (Lama pacos) brainstem and colocalization with CGRP. J Chem Neuroanat 2011;41:63-72.

10. Coveñas R, Mangas A, Medina LE, Sánchez ML, Aguilar LA, Díaz-Cabiale Z, et al. Mapping of somatostatin-28 (1-12) in the alpaca diencephalon. J Chem Neuroanat 2011;42:89-98.

11. Marcos P, Arroyo-Jiménez MM, Lozano G, González-Fuentes J, Lagartos-Donate MJ, Aguilar LA, et al. Mapping of tyrosine hydroxylase in the diencephalon of alpaca (Lama pacos) and co-distribution with somatostatin-28 (1-12). J Chem Neuroanat 2013;50-51:66-74.

12. Manger PR. Establishing order at the systems level in mammalian brain evolution. Brain Res Bull 2005;66:282-9.

13. Badlangana NL, Bhagwandin A, Fuxe K, Manger PR. Distribution and morphology of putative catecholaminergic and serotonergic neurons in the medulla oblongata of a subadult giraffe, Giraffa camelopardalis. J Chem Neuroanat 2007;34:69-79.

14. Bux F, Bhagwandin A, Fuxe K, Manger PR. Organization of cholinergic, putative catecholaminergic and serotonergic nuclei in the diencephalon, midbrain and pons of sub-adult male giraffes. J Chem Neuroanat 2010;39:189-203.

15. Ferreira G, Meurisse M, Tillet Y, Lévy F. Distribution and colocalization of choline acetyltransferase and P75 neurotrophin receptors in the sheep basal forebrain: implications of the use of a specific cholinergic immunotoxin. Neuroscience 2001;104:419-39.

16. Armstrong DM, Saper CB, Levey AI, Wainer BH, Terry RD. Distribution of cholinergic neurons in rat brain: demonstrated by the immunocytochemical localization of choline acetyltransferase. J Comp Neurol 1986;216:53-68.

17. Dell LA, Gruger JL, Bhagwandin A, Jillani NE, Pettigrew JD, Manger PR. Nuclear organization of cholinergic, putative catecholaminergic, and serotonergic systems in the brains of two megachiropteran species. J Chem Neuroanat 2010;40:177-95.

18. Cortés R, Arvidsson U, Schalling M, Ceccatelli S, Hökfelt T. In situ hybridization studies on mRNAs for cholecystokinin, calcitonin gene-related peptide and choline acetyltransferase in the lower brain stem, spinal cord and dorsal root ganglia of rat and guinea pig with special reference to motoneurons. J Chem Neuroanat 1990;3 467-85.

19. Henderson Z. Overlap in the distribution of cholinergic and catecholaminergic neurons in the upper brainstem of the ferret. J Comp Neurol 1987;265:581-92.

20. Henderson Z, Sherriff FE. Distribution of choline acetyltransferase immunoreactive axons and terminals in the rat and ferret brainstem. J Comp Neurol 1991;314:147-63.

21. Houser CR, Crawford GD, Barber RP, Salvaterra PM, Vaughn JE. Organization and morphological characteristics of cholinergic neurons: an immunocytochemical study with a monoclonal antibody to choline acetyltransferase. Brain Res 1983;266:97-119.

22. Jones BE, Beaudet A. Distribution of acetylcholine and cate- 
cholamine neurons in the cat brainstem: a choline acetyltransferase and tyrosine hydroxylase immunohistochemical study. J Comp Neurol 1987;261:15-32.

23. Kimura H, Maeda T. Aminergic and cholinergic systems in the dorsolateral pontine tegmentum. Brain Res Bull 1982;9:493-9.

24. Levey AI, Wainer BH, Mufson EJ, Mesulam MM. Co-localization of acetylcholinesterase and choline acetyltransferase in the rat cerebrum. Neuroscience 1983;9:9-22.

25. Manaye KF, Zweig R, Wu D, Hersh LB, de Lacalle S, Saper $\mathrm{CB}$, et al. Quantification of cholinergic and select non-cholinergic mesopontine neuronal populations in the human brain. Neuroscience 1999;89:759-70.

26. Marcos P, Coveñas R, Neuroanatomical relationship between the cholinergic and tachykininergic systems in the adult human brainstem: an immunohistochemical study. J Chem Neuroanat 2019;102:101701.

27. Mesulam MM. Cholinergic pathways and the ascending reticular activating system of the human brain. Ann NY Acad Sci 1995;757:169-79.

28. Motts SD, Slusarczyk AS, Sowick CS, Schofield BR. Distribution of cholinergic cells in guinea pig brainstem. Neuroscience 2008;154:186-96.

29. Nakamura Y, Hassler R, Kataoka K, Bak IJ, Kim JS. Regional distribution of choline acetyltransferase and acetylcholinesterase activity in baboon brain. Folia Psychiatr Neurol Jpn 1976;30:186-94.

30. Oda Y. Choline acetyltransferase: the structure, distribution and pathologic changes in the central nervous system. Pathol Int 1999;49:921-37.

31. Oda Y, Nakanishi I. The distribution of cholinergic neurons in the human central nervous system. Histol Histopathol 2000;15:825-34.

32. Sakai K, Luppi PH, Salvert D, Kimura H, Maeda T, Jouvet M. Localization of cholinergic neurons in the cat lower brainstem. C R Acad Sci III 1986;303:317-24.

33. Satoh K, Fibiger HC. Distribution of central cholinergic neurons in the baboon (Papio papio). I. General morphology. J Comp Neurol 1985;236:197-214.

34. Satoh K, Fibiger HC. Distribution of central cholinergic neurons in the baboon (Papio papio). II. A topographic atlas correlated with catecholamine neurons. J Comp Neurol 1985;236:215-33.
35. Shiromani PJ, Armstrong DM, Berkowitz A, Jeste DV, Gillin JC. Distribution of choline acetyltransferase immunoreactive somata in the feline brainstem: implications for REM sleep generation. Sleep 1988;11:1-16.

36. Tago H, McGeer PL, McGeer EG, Akiyama H, Hersh LB. Distribution of choline acetyltransferase immunopositive structures in the rat brainstem. Brain Res 1989;495:271-97.

37. Yasuhara O, Aimi Y, Matsuo A, Kimura H. Distribution of a splice variant of choline acetyltransferase in the trigeminal ganglion and brainstem of the rat: comparison with calcitonin gene-related peptide and substance P. J Comp Neurol 2008;509:436-48.

38. Batten TF, Lo VK, Maqbool A, McWilliam PN. Distribution of calcitonin gene-related peptide-like immunoreactivity in the medulla oblongata of the cat, in relation to choline acetyltransferase-immunoreactive motoneurones and substance Pimmunoreactive fibres. J Chem Neuroanat 1989;2:163-76.

39. Marcos P, Corio M, Dubourg P, Tramu G. Reciprocal synaptic connections between neurotensin- and tyrosine hydroxylaseimmunoreactive neurons in the mediobasal hypothalamus of the guinea pig. Brain Res 1996;715:63-70.

40. Mesulam MM, Geula C, Bothwell MA, Hersh LB. Human reticular formation: cholinergic neurons of the pedunculopontine and laterodorsal tegmental nuclei and some cytochemical comparisons to forebrain cholinergic neurons. J Comp Neurol 1989;283:611-33.

41. Ichikawa T, Ajiki K, Matsuura J, Misawa H. Localization of two cholinergic markers, choline acetyltransferase and vesicular acetylcholine transporter in the central nervous system of the rat: in situ hybridization histochemistry and immunohistochemistry. J Chem Neuroanat 1997;13:23-39.

42. Trifonov S, Houtani T, Hamada S, Kase M, Maruyama M, Sugimoto T. In situ hybridization study of the distribution of choline acetyltransferase mRNA and its splice variants in the mouse brain and spinal cord. Neuroscience 2009;159:344-57.

43. Lévy F, Meurisse M, Ferreira G, Thibault J, Tillet Y. Afferents to the rostral olfactory bulb in sheep with special emphasis in the cholinergic, noradrenergic and serotonergic connections. J Chem Neuroanat 1999;16:245-63.

44. Mena-Segovia J. Structural and functional considerations of the cholinergic brainstem. J Neural Transm 2016;123:731-6.

Received for publication: 21 April 2021. Accepted for publication: 4 June 2021.

This work is licensed under a Creative Commons Attribution-NonCommercial 4.0 International License (CC BY-NC 4.0).

(C) Copyright: the Author(s), 2021

Licensee PAGEPress, Italy

European Journal of Histochemistry 2021; 65(s1):3266

doi:10.4081/ejh.2021.3266 